\title{
TnphoA and Its Use in Transposon Mutagenesis
}

\author{
D. K. Das ${ }^{1^{*}}$, E. W. Nester ${ }^{2}$ \\ ${ }^{1}$ Post Graduate Department of Biotechnology, T. M. Bhagalpur University, Bhagalpur, India \\ ${ }^{2}$ Department of Microbiology, School of Medicine, University of Washington, Seattle, USA \\ Email: *dilipdas1@live.com
}

Received September 23, 2013; revised October 23, 2013; accepted October 30, 2013

Copyright (C 2014 D. K. Das, E. W. Nester. This is an open access article distributed under the Creative Commons Attribution License, which permits unrestricted use, distribution, and reproduction in any medium, provided the original work is properly cited. In accordance of the Creative Commons Attribution License all Copyrights @ 2014 are reserved for SCIRP and the owner of the intellectual property D. K. Das, E. W. Nester. All Copyright ( 2014 are guarded by law and by SCIRP as a guardian.

\section{ABSTRACT}

TnphoA construct was derived from the transposon Tn5, which contained a kanamycin resistance gene flanked by two IS50 elements. This construct contains a version of the alkaline phosphatase gene with its signal sequence and promoter deleted, which will result in a blue colony phenotype on X-phos (5-bromo-4-chloro-3-indolyl phosphate toluidine salt) containing media when secreted into the periplasm. This secretion can occur only if the TnphoA has been inserted into a gene with a signal sequence which directs that gene-product to leave the cytosol. Additionally, the transposon must be inserted into the correct orientation and the same reading frame as the gene has been inserted into. TnphoA's transposition activity derives from that of this Tn5 transposon by a conservative mechanism in a relatively rare but highly regulated process. The phoA portion of the TnphoA construct came from Escherichia coli K12 where it coded for the periplasmic protein alkaline phosphatase. This enzyme must be secreted from the cytoplasm in order to demonstrate activity and its secretion is determined by the presence of a signal sequence at its amino-terminal end. TnphoA mutagenesis is the identification of new genes that code for transmembrane or secreted proteins. TnphoA is an extremely useful construct, which along with other derivatives of the transposon Tn5 is used extensively in transposon mutagenesis based genetic analysis. TnphoA will ensure its continued significance and prominence in the area of transposon mutagenesis. TnphoA has been used to isolate new chromosomal genes, whose products are secreted, as in the case of some virulence genes in Agrobacterium tumefaciens and symbiotic genes in Rhizobium meliloti.

\section{KEYWORDS}

TnphoA Mutagenesis; Agrobacterium tumefaciens; Transposition; Transmembrane Proteins; Fusion Proteins; Tranposase Protein

\section{Introduction}

In this study of protein export, protein fusions, usually containing the cystol-bound enzyme $\beta$-galactosidase, have been central players. In order to advance studies involving protein fusions, the TnphoA construct was derived from the transposon $\mathrm{Tn} 5$, which contained a kanamycin resistance gene flanked by two IS50 elements. This construct contains a version of the alkaline phosphatase gene with its signal sequence and promoter deleted, which will result in a blue colony phenotype on X-phos (5-bromo-4chloro-3-indolyl phosphate toluidine salt) containing media when secreted into the periplasm. This secretion can

*Corresponding author. occur only if the TnphoA has been inserted into a gene with a signal sequence which directs that gene-product to leave the cytosol. Additionally, the transposon must be inserted into the correct orientation and the same reading frame as the gene has been inserted into.

This construct can be used as a tool in transposon mutagenesis to isolate and characterize genes that give rise to proteins with domains reaching beyond the cell's inner membrane. It can also be used to do studies on the transmembrane topology of these proteins. TnphoA could also have utility in determining what precise signals are involved in a protein's export if those signals exist within the protein itself. However, there are limitations to the application of TnphoA as a tool of molecular biology. For 
example, many gram negative bacterial species possess endogenous expression of alkaline phosphatase and this makes it impossible to distinguish mutagenesis from nonmutagenised colonies since all will appear blue on indicator media. This difficulty can be overcome by first making phoA minus mutants.

Regardless of any limitations, TnphoA has proven to be an extremely useful tool in genetic analysis, particularly because it occurs in the context of numerous other Tn5 derivatives that can complement each other and add to each other's utility. One of those Tn5 derivatives, Tnlacz, can be used in conjunction with TnphoA in localizing a protein's normal location within a cell. Mini-Tn5 transposons such as mini-Tn5 lacz2, mini-Tn5 phoA, miniTn5 luxAB also help to give the researcher a more complete set of molecular biology tools to work within the context of transposon mutagenesis. Plasposons, which contain elements of both transposons and plasmids, also help to round out the full range of tools that can be used to extend TnphoA's usefulness.

\section{Derivation of TinphoA}

To construct TnphoA [1] first inserted phoA sequences from plasmid CH39 into IS50 from plasmid RI122. This was done by treating pRI122 with Xba I, Bal31 exonuclease and Xho I and by treating pCH39 with Pst I, $\mathrm{T}_{4} \mathrm{DNA}$ ligase was used to seal the nicks [1]. This newly made phoA-IS50 ${ }_{\mathrm{R}}$ derivative was then permitted to recombine with the complete Tn5 from lamda 431 giving TnphoA [1]. TnphoA was then allowed to transpose into an F' factor which in turn inserted into plasmid pBR 322 from which restriction mapping was performed [1].

An opal (UGA) nonsense codon was found in this construct in the IS50, region which prevented expression of phoA except in a strain with an opal suppressing mutation [1]. The opal codon was eliminated from TnphoA through the use of a mutator strain (CC130) which resulted in an A-to-G change at the critical position in IS50 [1]. The transposon from which this opal codon was eliminated is now called TnphoA whereas the original is known as TnphoA (op). This change allows for phoA expression and hence for the blue colony phenotype in cases where the transposon inserts in the correct orientation and the phoA gene is in the same reading frame as a gene with a signal sequence and promoter.

\section{Mechanism of TnphoA Action}

\subsection{Tn5 Transposition Process}

TnphoA's transposition activity derives from that of this Tn5 transposon by a conservative mechanism in a relatively rare but highly regulated process [2]. The required elements for $\operatorname{Tn} 5$ transposition are the transposase protein (encoded from the transposase gene on the Tn5 it- self), specific end sequences (which define the termini of the transposon), and the target DNA [3]. Host proteins and conditions, such as the degree of DNA dam methylation, also play a role in the regulation and frequency of transposition, as does the transposon-encoded Inh (Inhibitor) protein.

Although the exact mechanism of Tn5 transposition is not completely know. The following model is useful for understanding the system. First, there is sequence-specific binding of the transposase protein to the 19 bp specific end sequences of Tn5 [3]. A synaptic complex then forms in which the ends of the transposon are brought together by transposase oligomerization [3]. Cleavage of the DNA adjacent to the transposon is mediated by the transposase protein when then binds the target DNA sequence [3]. Next both strands of the target DNA are cleaved and strand become covalently linked to the target's 5'- $\mathrm{PO}_{4}$ ends [3]. Lastly there is a repair process which resolves the transposition [3].

\subsection{Mechanism Underlying Expression of Alkaline Phophatase Activity}

The phoA portion of the TnphoA construct came from Escherichia coli K12 where it codes for the periplasmic protein alkaline phosphatase [4]. This enzyme must be secreted from the cytoplasm in order to demonstrate activity and its secretion is determined by the presence of a signal sequence at its amino-terminal end [4]. Once the promoter and signal sequence are deleted from the phoA gene, its activity can only be detected if its transcription is placed under the control of (and in the same reading frame as) another gene's promoter that has a signal sequence at its amino terminus. This effectively makes TnphoA a probe for export signals in proteins into whose genes it inserts [1].

In order to confirm that the signal sequence of the other protein in the fusion is required to obtain alkaline phosphatase activity, an experiment was performed by [1] in their original paper on the construction of TnphoA. A deletion mutant of bla (the gene which codes for $\beta$-lactamase), missing the signal sequence, had been previously constructed [5]. Insertion into the wild type bla gene resulted in alkaline phosphatase activity whereas insertion into the deletion mutant of bla did not. Therefore, the activity of the bla-phoA hybrid protein clearly depends on the existence of this signal sequence in $\beta$ lactamase [1].

Other experimental data also points to the utility of the TnphoA construct and confirms our understanding of its mechanism of action. For example, approximately 75\% of 57 independently isolated insertions were located in different positions of the target DNA (shown by restriction enzyme analysis) [1]. This suggests that TnphoA maintains the same low insertional specificity of Tn5. 
Additionally; cell fractionation confirmed that hybrid proteins located to the cell periplasm [1]. Finally, it was also shown that the hybrid proteins possessed substantial stability (with a half life of 30 mutants in the case of the bla-phoA fusions). Although most eventually broke down to give alkaline phosphatase-sized fragments [1].

\section{Applications of TnphoA Mutagenesis}

\subsection{Overview}

Several applications of TnphoA mutagenesis have already been outlined in brief. More thorough descriptions in the contexts of specific examples will now be explored. The ability of TnphoA to give insights into the membrane topology structure of proteins will be addressed with consideration given to Tnlacz-another Tn5 derivative, which is complementary to TnphoA. Along similar lines, an example of protein characterization in Haemophilus influenza will be described. Finally, the utilization of TnphoA mutagenesis to identify new virulence genes in Agrobacterium tumefaciens will also be reviewed briefly.

\subsection{Analyzing Membrane Protein Topology with TnphoA}

Since the enzymatic activity of alkaline phosphatase depends on its subcellular location, with export to the periplasm corresponding to high levels of activity and presence in the cytoplasm corresponding to low levels of activity, this can be a useful tool to analyze membrane protein topology. Other methods to ascertain information about membrane protein structure do exist, such as applying proteases or other reactive molecules, but this genetic approach has the advantage of not depending directly on exposure to amino acid side chains [1].

One example of how this approach has been successfully used can be found in the case of bex locus in encapsulated Haemophilus influenzae. A $5.1 \mathrm{~kb}$ region in the capsulation locus of $H$. influenzae was determined using Sanger dideoxy-sequencing and found to contain four open reading frames, called bexd, bexC, bexB, and bexA [6]. These genes (excluding the 3'-end of bexD) were cloned into a plasmid, pLNB24, and mutagenized with TnphoA [6]. Mutants were selected through their production of the blue-colony phenotype on X-phos media and were then examined further [6]. All three of the clones that were looked at were located in the 5' half of bexC, suggesting that its gene product is secreted into the periplasm [6]. Based on this and other information it was postulated that this protein may participate in a pore or carrier mechanism which might assist in the establishment and maintenance of the polysaccharide capsule of $H$. influenzae, which is a major virulence determinant [6].

A major advance in membrane protein topology studies came with the development by [7] of Tnlacz, which is also a Tn5 derivative and complementary to TnphoA. The lacz gene codes for the enzyme $\beta$-galactosidase which is only detectable in the cytoplasm, meaning that Tnlacz must insert into a gene which is not secreted from the cytosol frequently substantial in order to be detected. Even if the Tnlacz inserts into a different reading frame from the gene it has inserted into, there is still frequently substantial $\beta$-galactosidase activity, possibly due to translational [7]. Due to its complementary function with TnphoA, the Tnlacz construct can aid in the determination of where a protein or protein domain is normally within the cell. This was shown by [7] with the pBR325 plasmid which encodes chloramphenicol transacetylase, $\beta$-lactamase and tetracycline resistance protein [7].

Further enhancing the utility of the TnphoA and Tnlacz contructs is the ability to convert one type of fusion to another through a process known as fusion switching which uses recombination or in vitro manipulation [7]. This is possible because both constructs share the same $48 \mathrm{bp}$ left-end sequence from the Tn 5 transposon from which they were each derived. Since it is possible to generate alkaline phosphatase and $\beta$-galactosidase fusions at the same location in a protein, a comparison of their enzymatic activities can be performed to better establish the subcellular location of the protein site [7].

\subsection{Identification of Agrobacterium tumefaciens Virulence Genes}

One of the major applications of TnphoA mutagenesis is the identification of new genes that code for transmembrane or secreted proteins. An excellent example of this application is found in the case of Agrobacterium tumefaciens, gram negative bacteria that causes crown gall tumors on plants through a unique DNA transfer process [8] undertook an extensive mutational analysis of the organism using TnphoA mutagenesis. Advantages of this approach included the transposon's (much like other derivatives of Tn5) relatively random insertions into the genome, its relatively high degree of stability and the likelihood that may genes involved in the transfer process code for transmembrane or periplasmic proteins.

Before the transposon mutagenesis could begin, however, it was first necessary to derive phoA minus mutants. This was because $A$. tumefaciens expresses endogenous alkaline phosphatase activity that would completely mask the mutant phenotype. Chemical mutagenesis was used successfully to obtain multiple strains which had stable pho minus phenotypes [8] and continued virulence was confirmed to ensure that virulence related genes remained functional [8].

TnphoA was introduced into a phoA minus, virulent strain on a plsmid which is not maintained in A. tumefaciens [8]. The advantage of using such a "suicide" vector which, in this case, contains the transposase gene outside 
of the mobilized transposon, is that the likelihood of the insert being maintained at that site is increased since the transposase gene would be required for future transposition activity. This procedure was pioneered in Rhizobium (a relative of Agrobacterium) by [9]. Approximately 2000 mutants of $A$. tumefaciens with phoA activity were screened for virulence from which 30 avirulent or weakly virulent mutants were drawn [8]. Characterization of the mutants using Southern analysis showed which of the mutants located were previously known and which were newly identified genes [8].

\section{Discussion}

TnphoA is an extremely useful construct, which along with other derivatives of the transposon Tn5 is used extensively in transposon mutagenesis based genetic analysis. One important set of other Tn5 derivatives includes the mini-transposons, which include mini-Tn5 phoA as well as mini-Tn5lacz2 and mini-Tn5luxAB. Basically, these mini-transposons are much shorter (the miniTn5phoA for example is approximately half the size of the original TnphoA) because nonessential sequences have been trimmed off the transposon [10]. This includes the transposase gene itself which has been positioned outside the transposon's inverted repeats on the delivery suicide plasmid, though still cis to the transposon [10]. Two advantages of this approach, beyond the reduction in size of the transposon insert, are that it prevents further transposition and that it permits additional rounds of mini transposon mutagenesis because no immunity protein is present in the cell [11]. Whereas TnphoA and Tnlacz can be used to explore membrane protein topology, TnluxAB encodes bioluminescence and can be used to visualize bacterial presence for example in an inoculated Rhizobial strain [12].

Although not directly related to TnphoA mutagenesis, the TnMod plasposon rapid cloning system described by [11] can help extend TnphoA's usefulness by combining some of the best features of plasmids and transposons. TnMod, the basic plasposon, contains Tn5 inverted repeats, various restriction multiple cloning sites, antibiotic resistance cassettes that can be exchanged with one another, and a conditional origin of replication [11]. After TnMod insertional mutagenesis occurs, clone recovery is relatively simple and quick: genomic DNA is digested with an enzyme that cuts at one end of TnMod, the DNA is self-ligated, the ligation mixture is transformed into E.coli, and the cells are then selected for the antibiotic resistance encoded by TnMod [11]. Combining the advantages of this plasposon system with the advantages of TnphoA mutagenesis could be a fruitful avenue to explore in the future.

Protein fusions have been and will continue to be extensively used to study how and whether proteins are exported. Transposon mutagenesis is the case of TnphoA as well as certain other derivatives of Tn5 has added to the ways in which hybrid proteins can become tools of molecular biology. TnphoA has been used to isolate new chromosomal genes, whose products are secreted, as in the case of some virulence genes in Agrobacterium tumefaciens and symbiotic genes in Rhizobium meliloti. It has been used to help characterize the bexC gene in Haemophilus influenzae and to explore the topology of membrane proteins in conjunction with the Tnlacz construct. Improvements on the original TnphoA construct by [1] such as the miniTn5-phoA construct, which has the transposase gene in cis but external to the transposed element, have further enhanced the utility of this construct. Further variations and improvements to TnphoA will ensure its continued significance and prominence in the area of transposon mutagenesis.

\section{Conclusion}

TnPhoA which is derived from Tn5 is an important tool in molecular biology for transposon mutagenesis and also for isolation of new chromosomal genes such as virulence genes in Agrobacterium tumefaciens and symbiotic genes in Rhizobium meliloti. There are some minitransposons of TnPhoA such as mini-TnPhoA, mini-Tn5lacz and mini-Tn5luxAB which are small in size, can prevent further transposition and permit further rounds of minitransposon mutagenesis and can be used to explore membrane protein topology. Plasposons, which contain elements of both transposon and plasmids, also help to extend TnPhoA's usefulness.

\section{REFERENCES}

[1] C. Manoil and J. Beckwith, "TnphoA: A Transposon Probe for Protein Export Signals," Proceedings of the National Academy of Sciences of the USA, Vol. 82, No. 23, 1985, pp. 8129-8133. http://dx.doi.org/10.1073/pnas.82.23.8129

[2] W. S. Reznikoff, “The Tn5 Transposon,” Annual Review of Microbiology, Vol. 47, 1993, pp. 945-963. http://dx.doi.org/10.1146/annurev.mi.47.100193.004501

[3] I. Y. Goryshin and W. S. Reznikoff, “Tn5 in Vitro Transposition,” Journal of Biological Chemistry, Vol. 273, 1998, pp. 7367-7374. http://dx.doi.org/10.1074/jbc.273.13.7367

[4] C. S. Hoffman and A. Wright, "Fusions of Secreted Proteins to Alkaline Phosphatase: An Approach for Studying Protein Secretion," Proceedings of the National Academy of Sciences of the USA, Vol. 82, No. 15, 1985, pp. 51075111. http://dx.doi.org/10.1073/pnas.82.15.5107

[5] J. Kadonga, A. Gautier, D. Straus, A. Charles, M. Edge and J. Knowles, Journal of Biological Chemistry, Vol. 259, 1984, pp. 2149-2154.

[6] J. S. Kroll, B. Loynds, L. N. Brophy and E. R. Moxon, "The Bex Locus in Encapsulated Haemophilus influenze: A Chromosomal Region Involved in Capsule Polysaccha- 
ride Export,” Molecular Microbiology, Vol. 4, No. 11, 1990, pp. 1853-1862.

http://dx.doi.org/10.1111/j.1365-2958.1990.tb02034.x

[7] C. Manoil, "Analysis of Protein Localization by Use of Gene Fusions with Complementary Properties,” Journal of Bacteriology, Vol. 172, 1990, pp. 1035-1042.

[8] G. A. Cangelosi, E. A. Best, G. Martinetti and E. W. Nester, "Genetic Analysis of Agrobacterium," Methods in Enzymology, Vol. 204, 1991, pp. 384-397.

[9] S. Long, S. McCune and G. C. Walker, "Symbiotic Loci of Rhizobium meliloti Identified by Random TnphoA Mutagenesis,” Journal of Bacteriology, Vol. 170, 1988, pp. 4257-4265.

[10] V. de Lorenzo, M. Herrero, U. Jakubzik and K. N. Tim- mis, "Mini-Tn5 Transposon Derivatives for Insertion $\mathrm{Mu}$ tagenesis, Promoter Probing, and Chromosomal Insertion of Cloned DNA in Gram-Negative Eubacteria," Journal of Bacteriology, Vol. 172, 1990, pp. 6568-6572.

[11] J. J. Dennis and G. J. Zylstra, "Plasposons: Modular SelfCloning Minitransposon Derivatives for Rapid Genetic Analysis of Gram-negative Bacterial Genomes,” Applied and Environmental Microbiology, Vol. 64, 1998, pp. 2710 2715.

[12] R. Biovin, F. P. Chalifour and P. Dion, "Construction of a Tn5 Derivative Encoding Bioluminescence and Its Introduction in Pseudomonas, Agrobacterium and Rhizobium," Molecular and General Genetics, Vol. 213, 1988, pp. 5055. http://dx.doi.org/10.1007/BF00333397 